\title{
Recherche, ingénierie, création artistique : processus, prototypes, productions
}

\section{Résumé}

Dans cet article, nous abordons la question de la démarche recherche et création dans les collaborations artistes-chercheurs-ingénieurs. La perspective est double, reposant sur l'histoire (sont évoquées les collaborations protéiformes entre artistes, chercheurs et ingénieurs depuis 1950) et sur une étude de cas (le partenariat entre la compagnie de spectacle ALIS et l'Université de Technologie de Compiègne). Nous nous intéressons plus spécifiquement aux «technosciences », même si d'autres champs scientifiques sont concernés par ce type de collaborations.

Il ressort que ces collaborations invitent à réordonner les ordres de priorité, en valorisant le processus plutôt que le résultat, mais aussi en mettant en lumière le rôle du prototype comme objet commun aux artistes, aux chercheurs et aux ingénieurs. Apparaît également comme crucial le rôle du traducteur ou intermédiaire entre les différents mondes. Nous proposons une première modélisation à valeur heuristique du cadre et des enjeux de ces collaborations afin de mieux appréhender celles-ci.

\section{Mots-clés}

Recherche, ingénierie, création artistique, collaboration, processus, prototype, 9Evenings, Alis, UTC, écriture numérique.

\section{Notices biographiques}

Serge Bouchardon (utc.fr/ bouchard) est Professeur à l'Université de Technologie de Compiègne

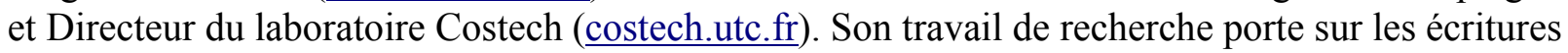
numériques, en particulier sur la littérature numérique.

Dernier livre paru : La valeur heuristique de la littérature numérique, Hermann, 2014.

Clarisse Bardiot est maître de conférences à l'université de Valenciennes et chercheur associée au CNRS (laboratoire Thalim/ARIAS). Ses travaux portent sur les digital performances, la documentation et la conservation des œuvres numériques, les relations artistes-ingénieurs et les humanités numériques.

Hélène Caubel est directrice de production dans le spectacle vivant. Elle accompagne notamment le travail de l'artiste Pierre Fourny, au sein d'ALIS, et y porte la dynamique de rapprochement artscience-éducation. 


\section{Recherche, ingénierie, création artistique : processus, prototypes, productions}

Introduire des artistes à l'université et dans des laboratoires de recherche, aux côtés d'ingénieurs et de chercheurs, n'est pas neutre. De l'accès (aux technologies) à l'excès (des disciplines): telle pourrait être résumée l'aventure de ces collaborations. D'un côté, cette introduction pose de nombreuses questions, notamment sur les fonctions sociales de l'artiste et de l'art. D'un autre côté, elle interroge les méthodologies propres à la recherche : quel contexte dessiner/designer pour favoriser l'innovation ? La venue de l'artiste dans des laboratoires de recherche, que ces derniers soient privés ou publics, est une réponse possible, tout particulièrement lorsqu'il s'agit de technologies numériques. Mais l'alchimie est fragile : le dépassement des disciplines (censé engendrer ruptures scientifiques, techniques et esthétiques) présuppose que l'art ne soit pas au service de la science et vice versa. Des projets hybrides, dont le statut épistémologique reste à définir, apparaissent.

Nous mettrons en regard une évocation de l'histoire de ces collaborations depuis 1950 (et ce qu'elle nous inspire) avec une étude de cas, celle du partenariat entre ALIS, compagnie de spectacle, et l'Université de Technologie de Compiègne.

\section{1 - Quelques jalons historiques pour une ébauche de typologie des collaborations artistes - ingénieurs - scientifiques}

\section{Réconcilier les « deux cultures »}

En 1959, le romancier et scientifique anglais C.P. Snow donne une conférence sur la scission entre « les deux cultures », à savoir les arts et les humanités d'un côté, et les sciences de l'autre (Snow, 1968). Il y déplore un divorce croissant et une incapacité à dialoguer. Pourtant, c'est dans cette même période que des liens entre art, science et technologie s'instaurent (Debatty et Grover, 2011). L'intervention de Snow, largement relayée et commentée (Lee, 2004 ; Goodyear, 2004), témoigne que cela ne va pas de soi. De part et d'autre de l'Atlantique, des entreprises, puis des universités, intègrent des artistes dans leurs programmes de recherche. Les modalités du dialogue sont à géométrie variable, depuis l'emploi des artistes dans des laboratoires aux résidences sur le long terme ou ponctuelles, avec une présence quotidienne aux côtés des chercheurs ou bien des rendez-vous plus hiératiques, chacun travaillant de son côté dans son propre environnement de travail. Le cas de figure où un chercheur séjourne dans l'atelier de l'artiste demeure une exception. Le trajet se fait beaucoup plus souvent depuis l'atelier vers le laboratoire que l'inverse ${ }^{1}$.

Dès le début des années 1960, les laboratoires Bell engagent des artistes dans certains programmes, de manière plus ou moins formelle : les compositeurs James Tenney et Jean-Claude

\footnotetext{
$1 \quad$ Un contre exemple serait Billy Klüver, ingénieur de Bell labs, travaillant au début des années 1960 avec et chez des artistes tel que Tinguely, Rauschenberg ou les chorégraphes de la Judson Church. Mais ceci se passait en général sur son temps personnel, et non dans le cadre du travail qu'il accomplissait alors pour Bell Labs.
} 
Risset aux côtés de Max Matthews (considéré comme le père de l'informatique musicale) ; les plasticiens Stan VanDerBeek et Lillian Schwartz avec l'ingénieur Ken Knowlton pour réaliser les premiers films en images de synthèse. Le point culminant de la collaboration artiste/ingénieur dans ces laboratoires est l'organisation, à l'initiative d'un ingénieur, Billy Klüver, et avec l'aide de Robert Rauschenberg, de la manifestation 9 Evenings, Theatre \& Engineering en 1966 (Bardiot, 2006). Lors de cet événement, une trentaine d'ingénieurs travaillent avec dix artistes (John Cage, Lucinda Childs, Öyvind Fahlström, Alex Hay, Deborah Hay, Steve Paxton, Yvonne Rainer, Robert Rauschenberg, David Tudor et Robert Whitman) pour créer dix performances qui mêlent arts de la scène et nouvelles technologies. Pour les artistes comme pour les ingénieurs, il s'agit dans la plupart des cas d'une première, ces deux mondes ne se côtoyant encore qu'exceptionnellement. Plusieurs configurations sont testées : le travail en binôme artisteingénieur, le recours à des spécialistes comme consultants, les rencontres informelles, le travail dissocié (au début, les ingénieurs travaillent dans les laboratoires ou chez eux, sans la présence des artistes) puis le travail conjoint dans un même espace-temps. Dans la foulée de 9 Evenings, Billy Klüver crée avec Fred Waldauher, Robert Rauschenberg et Robert Whitman Experiments in Art and Technology (E.A.T.). Cet organisme à but non lucratif, qui a compté plusieurs milliers de membres, a pour vocation le rapprochement entre disciplines artistiques, recherches scientifiques et entreprises.

Parallèlement, Maurice Tuchman, conservateur au Los Angeles County Museum of Art (LACMA) crée le programme Art and Technology (1967-1971) (Lacma, 1971). Le principe est de favoriser les échanges entre artistes et entreprises. Ces dernières peuvent contribuer au programme selon différentes modalités, du don financier à l'accueil en résidence d'un artiste au sein de l'entreprise. Le premier objectif est la réalisation d'une œuvre avec les moyens de l'entreprise. Le second, plus flou, est que l'entreprise « ne peut que tirer un bénéfice incommensurable, directement ou indirectement, de son exposition à des personnes créatives » (Ibid.). La principale motivation des nombreuses entreprises contribuant à ce programme est la possibilité d'acquérir gracieusement un exemplaire de l'œuvre réalisée. C'est ainsi que Claes Oldenburg effectue une résidence chez Walt Disney Productions, John Chamberlain chez RAND, Robert Whitman chez Philco-Ford, Richard Serra chez Kaiser Steel, etc. Le programme a été relancé en 2014, avec des collaborations annoncées avec Google, SpaceX, NVIDIA, ou encore Accenture.

En Europe également, les « deux cultures » tissent des liens. Chez Philips, aux Pays-Bas, des collaborations sont initiées dès les années 1950 avec des artistes : citons Cysp 1, sculpture cybernétique de Nicolas Schöffer en 1956 ou encore la création du Pavillon Philips en 1958 pour l'Exposition Universelle, avec Le Corbusier, Iannis Xenakis et Edgard Varèse. En Angleterre, le Artists' Placement Group, créé en 1966, souhaite littéralement « placer » des artistes dans des groupes industriels et faire en sorte qu'ils y soient embauchés au même titre que les autres salariés sur une longue période. Il s'agit ici bien plus de s'immerger que de proposer une collaboration, un échange de pratiques.

Très vite, ces différentes expérimentations trouvent des prolongements à la fois dans les institutions culturelles et au sein des universités avec la création de cursus spécifiques : apparition de lieux dédiés à la rencontre entre recherche scientifique et pratiques artistiques, tels que l'IRCAM en 1975 ou le ZKM en 1997 ; naissance des " médialabs », dont l'un des précurseurs est le Center for Advanced Visual Studies (CAVS) créé par György Kepes au MIT en 1967. Très 
influencé par le modèle du Bauhaus ${ }^{2}$, le CAVS accueille des artistes en résidence selon le principe $\mathrm{du}$ « fellowship ». En plus des collaborations avec les ingénieurs et les chercheurs, les artistes enseignent tandis que des étudiants participent aux projets. Sous la direction d'Otto Piene, le Master of Science in Visual Studies est créé en 1976, avec la participation d'autres sections du département d'architecture.

\section{Deux exemples francophones}

Aujourd'hui, alors que les questions d'innovation et de créativité sont centrales, que fabs labs, living labs, hackerspaces et makerspaces sont érigés en nouveaux modèles de production et de travail (Lallement, 2015), les collaborations entre artistes, chercheurs et ingénieurs se multiplient dans le cadre de programmes portés par des institutions culturelles, des laboratoires de recherche, des écoles d'art, des universités, des " grandes écoles », des fondations d'entreprise ou encore des activités de mécénat. S'il est impossible de les citer toutes ici, nous prendrons deux exemples francophones s'inscrivant dans des cadres pédagogiques, les initiatives anglo-saxonnes étant par ailleurs davantage documentées (Debatty et Grover, 2011 ; Butcher et Broeckmann, 2010).

Dès sa création, le Fresnoy, proclamé « Bauhaus de l'électronique », « Ircam des arts plastiques ", pose les questions des matériaux contemporains de la production artistique, de l'expérimentation, et partant des conditions de collaboration entre artistes, élèves-ingénieurs et chercheurs. La nature de cette collaboration, au gré des projets et des profils, a connu de multiples avatars. Au-delà des projets spécifiques devant se réaliser dans un temps court (entre 6 et 12 mois en moyenne), des collaborations récurrentes (avec l'Ircam, l'INRIA, l'équipe MINT de l'IRCICA...) instaurent une relation sur le long terme.

En Belgique, l'institut New Media Art Technology (Numediart), créé en 2007 à l'Université de Mons prend sa source dans une université scientifique dont la vocation est de former des ingénieurs. Tous les 3 mois, un appel à projet est lancé, auquel peuvent répondre des artistes : il leur est alors possible de collaborer avec des équipes de recherche pendant une période de 3 à 6 mois pour réaliser le prototype d'une œuvre ou d'une technologie.

Si les points de départ de ces deux derniers exemples sont aux antipodes (une école d'art et une école d'ingénieur), les questions de la collaboration se posent dans les mêmes termes et n'ont pas toujours donné lieu à des cadres juridiques concrets ${ }^{3}$ : quid de la propriété intellectuelle et du dépôt d'éventuels projets ? Comment s'assurer que les ingénieurs et les chercheurs ne sont pas instrumentalisés au service des artistes et vice-versa?

On le voit, les problématiques esquissées dès les années 1950 et 1960 demeurent similaires. Comme le notait déjà Billy Klüver en 1966 dans le programme de 9 Evenings, « cela n'a pas été aussi facile qu'il y paraît. Les artistes ont dû faire preuve d'énormément de patience face au rythme lent des ingénieurs. Et les ingénieurs ont dû se débrouiller avec le flou des artistes, ces derniers n'ayant rien à tenir dans leurs mains ou à travailler $»^{4}$.

\footnotetext{
2 Les avant-gardes européennes des années 1920 demeurent un arrière-plan fondamental pour ces différents projets.

Si le Fresnoy travaille à la rédaction d'une convention avec les laboratoires de recherche avec lesquels l'école collabore régulièrement, cette convention n'est pas encore signée au moment de la rédaction de cet article.

9 Evenings : Theatre and Engineering. Edited by Pontus Hultén and Frank Königsberg, New York, Experiments in Art and Technology, The Foundation for Contemporary Performance Arts, 1966.
} 


\section{Une ébauche de typologie des collaborations artistes-ingénieurs-chercheurs}

Au-delà des particularités liées au contexte, aux modalités et surtout aux facteurs humains (souvent déterminants), quels paramètres retenir pour caractériser les collaborations artistesingénieurs-chercheurs? Sans définir une typologie, voici plusieurs propositions de " curseurs » permettant d'esquisser les points principaux d'une géométrie variable :

- Format (collaboration informelle, résidence, emploi, commande)

- Durée (quelques jours à plusieurs années)

- Mode (collaboration fragmentée ou continue)

- Objectif processus/produit (importance mise sur le processus, le prototype ou le produit, c'est à dire la création d'une œuvre ou d'un objet technologique)

- Rapport des artistes aux sciences et des scientifiques aux arts (les ingénieurs et les chercheurs ont-ils des connaissances artistiques et vice-versa ; un cas de figure particulier est l'artiste-ingénieur réunis en une seule et même personne)

- Organisation (de l'espace/temps partagé à la collaboration à distance)

- Cadre (de l'absence de convention à un cadre juridique précis avec prise en compte des questions de propriété intellectuelle)

- Initiateur (pouvoirs publics, institution culturelle, université, entreprise, chercheur, ingénieur, artiste)

- Lieu (entreprise, université, institution culturelle, lieu privé)

- Présence ou absence d'un intermédiaire

Comme le constate Elie During (Madlener, 2010), " ce sont les positionnements intersectifs et les rapports d'empiètement réciproque qui sont peut-être les plus intéressants à étudier, puisqu'ils obligent chacun à se déporter vers d'autres logiques de production, sans pour autant annuler les différences bien réelles qui existent entre les pratiques (artistiques, scientifiques)... ». L'étude des intersections entre ces différents paramètres reste à faire. Elle permettrait peut-être de faire apparaître des modèles récurrents et donc de définir une typologie précise des collaborations artistes-ingénieurs.

Si ce champ reste ouvert, il est d'ores et déjà possible d'identifier une personne clé de ces dispositifs de collaboration, qui a tendance à disparaître des chroniques au profit de la mise en avant de l'artiste et/ou de l'ingénieur, et qui pourtant bien souvent détermine, ajuste ou influence les paramètres mêmes de ces collaborations polymorphes. Cette figure, c'est celle, essentielle, du traducteur, de l'intermédiaire, de l'interprète entre deux mondes qui, s'ils ont des points communs (par exemple la question de l'expérimentation), n'ont pas toujours les repères suffisants pour entrer en dialogue. Des ponts doivent être créés. Ainsi, pour 9 Evenings, l'ingénieur Herb Schneider, inquiet par la tournure que prenait les événements, prit en main la situation et s'autoattribua ce rôle (Bardiot et Morris, 2006) ; au LACMA, c'est Maurice Tuchman qui a décidé des «mariages » entre artistes et entreprises puis a veillé à leur bon déroulement; au Fresnoy, Eric Prigent, responsable pédagogique de la seconde année, et Laurent Grisoni, professeur de science informatique à Lille 1, veillent à ces questions essentielles de communication. 


\section{modalités de collaboration dans 9 Evenings}

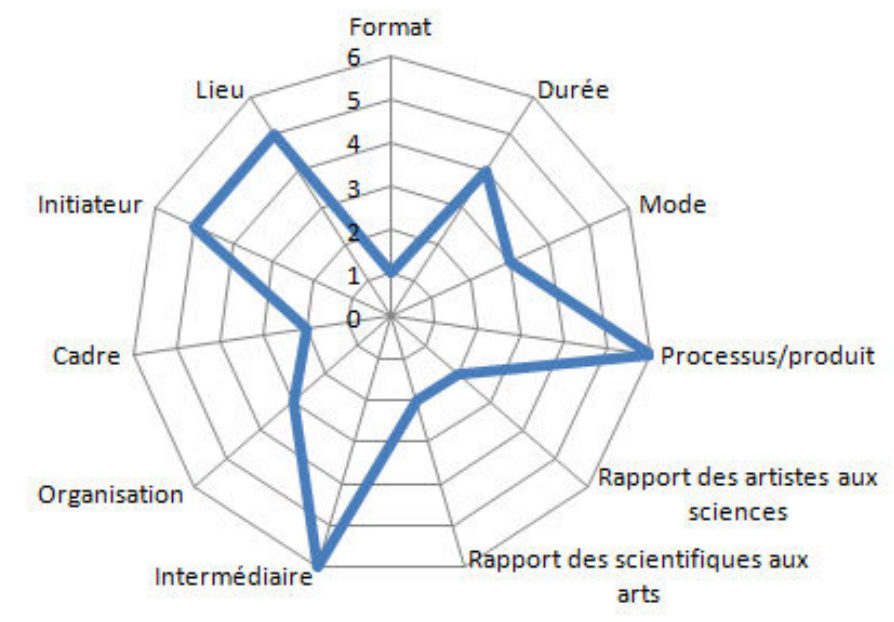

Légende : le graphe ci-dessus est pertinent dans une approche comparative et ne porte pas un système de valeurs. Son fonctionnement est visuel et non axiologique.

Format

Durée

Mode

Processus/produit

Rapport des artistes aux $0=$ aucun ; $3=$ éclairé $; 6=$ artiste-chercheur

sciences

Rapport des scientifiques $0=$ aucun ; $3=$ éclairé $; 6=$ chercheur artiste aux arts

Intermédiaire

Organisation

Cadre

Initiateur

Lieu

$0=$ processus $; 3=$ prototype $; 6=$ produit 4=chercheur ; $5=$ ingénieur ; $6=$ artiste 0=informelle ; $2=$ résidence $; 4=$ emploi ; 6=commande

$0=<1$ semaine $; 1=<1$ mois $; 2=<3$ mois; $3=<6$ mois; $4=<1$ an; $5=<2$ an; $6=>2$ ans

$0=$ très fragmenté $; 3=$ périodes de travail en commun ; $6=$ en continu

$0=$ absence $; 6=$ présence avec forte implication

$0=$ collaboration à distance $; 6=$ espace-temps partagé

$0=$ absence de convention; $6=$ cadre juridique précis

$0=$ pouvoirs publics ; $1=$ institution culturelle ; $2=$ université ; $3=$ entreprise ;

$0=$ lieu privé ; $1=$ institution culturelle ; $2=$ université ; $3=$ entreprise ; 6=hybride 
Des années 1950 à nos jours, les collaborations artistes-ingénieurs-chercheurs ne proposent pas un modèle univoque. La question de la production semblait un objectif essentiel dans les débuts : la collaboration avait lieu dans la perspective de la réalisation d'une œuvre, plus rarement d'une technologie. Pourtant, d'entrée de jeu, comme l'indique Maurice Tuchman plus haut, l'importance du processus est soulignée. Si bien que nombre de réflexions se sont orientées vers une mise en valeur des vertus du processus par rapport au résultat, à la qualité de l'œuvre produite (Butcher et Broeckmann, 2010). Celle-ci n'apparait plus alors parfois que comme un épiphénomène. Il existe un moyen-terme, entre le processus et la production : le prototype. C'est ce que souligne Elie During (Madlener, 2010) : "Comme le prototype industriel, le prototype artistique est un objet à la fois idéal (prospectif, projectif) et « expérimental » (à la fois inchoatif et indéterminé quant à ses fins, à ses usages). Du processus de création, il offre une stabilisation provisoire, mais cette première (ou n-ième) réalisation vaut tout de même démonstration : elle doit donner forme à une idée, elle doit l'exposer, autrement dit rendre sensible un ordre de possibilités opératoires. " Dans cette perspective, le prototype serait alors l'objet (à la fois objectif, méthodologie et production) commun aux artistes, aux chercheurs et aux ingénieurs, le lieu possible de la rencontre entre recherche (scientifique) et création (artistique), entre création (scientifique) et recherche (artistique).

\section{2 - Etude de cas : I'Université de Technologie de Compiègne et la compagnie ALIS}

\section{Les jalons de la rencontre : les notions de recherche et de création au sein de I'UTC et d'ALIS}

\section{La recherche, la science et la technologie au sein d'ALIS}

Dès ses origines en 1982, ALIS pratique la scène de façon singulière, cherchant sa voie dans l'invention de langages non verbaux. ALIS se tourne vers les objets du quotidien et les affiches publicitaires pour " prendre le plateau », et non vers les textes. S'il est question de rimes et de poésies, elles sont d'abord visuelles et viennent bousculer notre rapport au Verbe. À l'aube des années 2000, Pierre Fourny, fondateur d'ALIS, découvre des procédés graphiques et sémantiques originaux, qu'il nomme la Poésie à 2 mi-mots $^{5}$ et qu'il ne cesse depuis de faire proliférer, grâce à la puissance combinatoire de l'informatique. Le genre non 《répertorié » d'ALIS et la découverte de procédés inédits consacre la compagnie comme espace « exploratoire ». Même les sources d'inspiration revendiquées par Pierre Fourny (mis à part le performer Stuart Sherman), sont davantage à trouver du côté de l'interprétation de ses lectures scientifiques (dont Marshall McLuhan, Roland Barthes, Jacques Lacan...) que du côté des mondes de l'art.

Mais si ALIS a emprunté des chemins de traverse, elle s'est toujours servi des techniques et technologies à la disposition de tous, et n'a pas cherché à déployer une ingénierie spécifique en ce domaine. En ce sens, elle reste du côté des «solutions grand public», sans revendication esthétique particulière, davantage dans une recherche de maîtrise de sa propre production.

5 http://www.alis-fr.com/site/?q=node/26 


\section{L'importance du faire pour comprendre à l'UTC}

L'UTC est une école d'ingénieurs. Une réflexion y a été menée en 2014 par le Conseil Scientifique sur la recherche technologique, celle-ci étant de nature à qualifier et fédérer l'ensemble de la recherche qui s'y déploie.

La recherche technologique est une manière particulière de faire de la recherche scientifique. Elle conjugue trois dimensions en interaction :

- une dimension académique : elle génère des connaissances nouvelles et est soumise aux mêmes exigences académiques que n'importe quelle autre recherche scientifique,

- une dimension de pertinence sociétale : elle est soumise à des stimulations/pressions positives de la part du monde socio-économique ; elle y répond par de nouveaux dispositifs dont les usages peuvent être innovants,

- une dimension liée au système technique : elle nécessite de modéliser, concevoir, réaliser et évaluer des dispositifs, pas uniquement pour valider, mais également pour comprendre.

La recherche technologique procède ainsi non pas seulement d'un comprendre pour faire, mais aussi d'un faire pour comprendre : dans la recherche technologique, c'est aussi à partir de la réalisation de dispositifs ou procédés techniques que peut se créer de la connaissance scientifique.

Vis-à-vis des technologies, notamment numériques, les SHS sont en général envisagées soit avec une - indispensable - posture critique, soit dans une perspective d'acceptabilité sociale du changement technique. Une troisième voie peut être envisagée, permettant d'articuler ces deux approches : celle de la conception. Les outils et médiations sont dès lors abordés à la fois comme objet (matériel, numérique, organisationnel) constitué, fabriqué, partagé, et comme objet constituant, saisi, approprié, rendant possible les connaissances et activités individuelles et collectives. A travers une activité de conception, il s'agit pour les chercheurs de comprendre comment les techniques peuvent être constitutives / constituantes des expériences humaines et des pratiques sociales (Steiner, 2010).

Le prototype, en tant qu'objet intermédiaire, permet alors de mettre le doigt sur cette constitutivité technique, c'est-à-dire de comprendre comment les outils, les organisations matérielles, les technologies et systèmes d'information et de communication que nous concevons, développons et utilisons peuvent affecter nos façons de percevoir, de mémoriser, de raisonner, de définir des valeurs, des appartenances, mais aussi nos modes de rencontre, nos modalités d'interaction et nos manières d'être et d'agir ensemble.

\section{Quelle place pour la création artistique à l'UTC?}

L'art et la création, à l'UTC, s'enracinent dans la dynamique de recherche technologique et la fécondent. C'est un enjeu important pour une école d'ingénieurs. Au niveau de la formation, l'apprentissage de la créativité passe en effet aussi par la sensibilisation à des pratiques artistiques, qui détournent des usages et inventent des possibles. On peut d'ailleurs noter des similitudes entre la figure de l'ingénieur et celle de l'artiste. La démarche de conception de l'ingénieur relève d'un bricolage d'ingrédients hétérogènes (scientifiques, mais également sociaux, économiques) et se rapproche, comme tel, de l'activité de l'artiste (Odin et Thuderoz, 2010).

$\mathrm{Au}$ niveau de la recherche, il y a un intérêt fort à faire venir des artistes dans les laboratoires car ils créent, comme les scientifiques, des expériences. Vivre une expérience artistique comme expérience scientifique, c'est admettre qu'un vécu expérientiel peut avoir une 
dimension scientifique. Le geste de création permet de créer les conditions d'observabilité de la manifestation d'un phénomène. Le croisement entre arts, sciences et technologies permet alors de mettre en avant une démarche de recherche et création. Ce croisement peut aboutir à des réalisations innovantes, comme ce fut le cas pour le projet Immersive Music Painter ${ }^{6}$.

\section{Naissance et développement du projet La séparation}

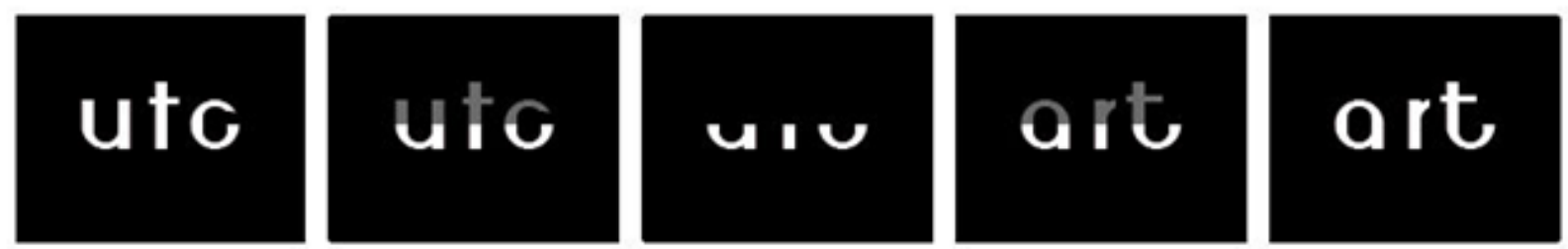

Captures d'une animation vidéo réalisée par Pierre Fourny permettant de faire émerger du mot "utc" le mot "art", les deux mots partageant la même moitié basse.

\section{Du festival Les Composites à l'appel à candidature du Centre d'innovation}

Le 3 avril 2012, dans le cadre du festival Les Composites, Pierre Fourny engage un dialogue avec Serge Bouchardon, enseignant et chercheur à l'UTC sur les écritures multimédias et auteur numérique au sein du collectif $i$-Trace, qui présente sa création Déprise. Serge Bouchardon invite alors Pierre Fourny à intervenir dans l'atelier du mineur Technologies Culturelles Numériques $^{7}$. La démonstration de Poésie à 2 mi-mots que l'artiste fera, le 13 novembre 2013, sous le titre "La Poésie à 2 mi-mots : codage numérique (2 signes) du code alphabétique (26 signes)" stimulera l'imagination et l'esprit critique de certains élèves-ingénieurs. Elle achèvera de convaincre Serge Bouchardon de l'opportunité qu'il y aurait à développer un projet de recherchecréation avec ALIS. Cela relancera aussi le projet de participer au quatrième " concours de labellisation 2012 de projets innovants » porté par le Centre d'innovation de l'UTC pour offrir un cadre à cette collaboration avec des perspectives de financement et d'aide personnalisée.

Après confirmation de l'éligibilité d'une démarche à fort caractère artistique, et malgré les débouchés industriels que doivent permettre les projets soumis, décision est donc prise de candidater pour un projet désormais intitulé La séparation. Le 24 décembre 2014, il sera labellisé par le Centre d'innovation qui en souligne la qualité, mais son financement est soumis à conditions : la maturation du projet doit être réalisée dans un objectif d'exploitation de l'innovation sur le marché; les aspects juridiques et de propriété intellectuelle doivent être clairement définis vis-à-vis de l'UTC, le porteur de projet étant en partie extérieur à l'université. Plusieurs réunions seront organisées pour définir les moyens de conformer La séparation aux conditions du Centre d'innovation, sans résultat concret pour le moment. Par ailleurs, il ne paraîtra pas opportun à l'équipe d'ALIS de se tourner vers l'Espace Jean Legendre n'ayant pas, à ce stade, de «productions à vendre ». C'est donc sur les désirs et motivations d'ALIS et de Serge

https://www.youtube.com/watch?v=dRlznjKKjB0

Un mineur est un diplôme d'établissement délivré par l'UTC, qui vient compléter la formation d'ingénieur dispensée. 
Bouchardon, sur la reconnaissance symbolique que confère l'UTC et grâce aux partenaires institutionnels ${ }^{8}$ de la compagnie de spectacle que la collaboration va se développer.

\section{Les conditions d'exercice et de financement... un hors piste assumé}

Serge Bouchardon fait une place à ALIS, au sein de l'UTC, en jouant un rôle d'intermédiaire et en impliquant autour de la Poésie à 2 mi-mots ses propres étudiants ainsi que des enseignants-chercheurs évoluant à l'intérieur ou l'extérieur de l'UTC. Ainsi, de mars 2013 à décembre 2014, des membres d'ALIS sont appelés à intervenir sur les sites de l'université à Compiègne. Ils y participent à des réunions de travail ou co-dirigent, en lien avec Serge Bouchardon, des élèves-ingénieurs qui se lancent dans le développement de logiciels et d'applications, au service de la Poésie à 2 mi-mots, dans le cadre des différentes « unités de valeur » dispensées à l'UTC ou de travaux personnels validés comme " unités de valeur », à savoir : écriture interactive et multimédia (SI28), ingénierie des systèmes interactifs (NF28), étude expérimentale (TX), réalisation de projet $(\mathrm{PR})$.

ALIS cherchera à inviter tout ce petit monde dans ses propres ateliers et bureaux, mais sans succès, du fait de leur éloignement de Compiègne et de la prégnance du cadre universitaire, qui est celui où tous les acteurs du projet se retrouvent : les étudiants car ils y suivent et y valident leur cursus, les enseignants-chercheurs car il s'agit bien évidemment de leur milieu professionnel, et l'équipe d'ALIS car elle y trouve une forme de légitimation et y découvre de nouvelles opportunités de circulation/diffusion.

Serge Bouchardon va entraîner l'équipe dans les réseaux universitaires internationaux de la littérature et de la poésie numériques qu'il fréquente en tant que chercheur-auteur et plus marginalement dans le secteur de l'éducation nationale où il est reconnu pour son expertise liée à l'enseignement de l'écriture numérique et pour ses créations.

Dans cette collaboration, ALIS trouve matière à affirmer son identité de "compagnielaboratoire". Valoriser cette identité et façon de faire de l'art en allant voir ailleurs auprès de ses partenaires historiques est à la fois une opportunité et une gageure. Du côté de l'université, Serge Bouchardon fait place à l'intégrité d'un travail artistique (dans ses doutes, tâtonnements, franches singularités...) dans un contexte en définitive peu favorable, y compris jusque dans les lieux d'art.

\section{La place des étudiants... point de bascule}

C'est en grande partie dans le frottement aux étudiants, mais aussi dans la reconnaissance de ce qu'ils sont en mesure de « donner » que le projet La séparation va trouver sa voie.

La demande que Pierre Fourny formule en direction des étudiants, à l'invitation de Serge Bouchardon, se heurtera à la connaissance empirique qu'il a de sa propre pratique de la Poésie à 2 mi-mots. La matière cumulée par l'artiste est dense et riche, mais elle est aussi éparse, et lacunaire d'un point de vue technique. Il faudra donc s'attacher à repasser par des chemins oubliés, en emprunter d'autres jamais arpentés, et à mettre en mots, pour pouvoir transmettre et avancer collectivement. Dans ces processus, Hélène Caubel, directrice de production d'ALIS, fait le lien entre toutes les parties impliquées; elle joue un rôle essentiel, celui de l'intermédiaire, en

$8 \quad$ La DRAC Picardie, le Conseil régional de Picardie, le Conseil général de l'Aisne et la Ville de Fère-en-Tardenois. 
collaboration avec Serge Bouchardon qui assume ce rôle pour l'UTC. Pierre Fourny propose d'améliorer le logiciel qu'il a coutume d'utiliser pour travailler sa Poésie à 2 mi-mots, tandis que Serge Bouchardon espère voir naître une web application permettant de donner à voir des pièces interactives de Poésie à 2 mi-mots, voire d'en « fabriquer ». Face au temps d'appropriation par le groupe des enjeux de cette poésie, et face aux difficultés techniques qu'elle soulève et aux compétences fines et variées qu'elle réclame, aucun de ses objectifs n'a été atteint jusqu'ici, mais de nombreux échanges et tentatives ont permis de voir naître, au sein de l'UTC, deux prototypes (l'application pour tablettes et smartphones La séparation ${ }^{9}$ et l'application Kinect à 2 mi-mots) et d'en inspirer d'autres dont ALIS a confié le développement, qu'elle voulait rapide et "robuste", à un développeur professionnel.

\section{Vers une résidence d'artistes en recherche à l'UTC}

Fin 2014, ALIS a ressenti le besoin, au regard des actuels critères d'appréciation des compagnies professionnelles conventionnées par le Ministère de la Culture et de la Communication (nombre de cession de spectacles dans l'année, nombre de salariés employés, reconnaissance des lieux partenaires de la compagnie...), de marquer le caractère officiel de la collaboration avec l'UTC et d'affirmer justement avec elle d'autres critères de reconnaissance.

Ainsi les acteurs du projet La séparation écrivent une convention et demandent un soutien auprès de la DRAC au titre du dispositif de résidence artistique. Une circulaire de 2006 évoque la possibilité pour les compagnies d'être accueillies par des universités. Cette convention, la première du genre à l'UTC, doit permettre de formaliser une convention générique qui pourrait servir à d'autres équipes artistiques à terme. L'UTC a choisi de parler de résidence d'artistes en recherche, pour souligner que l'artiste peut être pleinement impliqué dans le travail de recherche scientifique; symétriquement, dans le projet $L a$ séparation, les scientifiques interviennent également en tant qu'acteurs dans la création artistique. C'est cette interpénétration des deux mondes, qui fait s'estomper les frontières entre les rôles, qui autorise un processus de collaboration par certains côtés atypique. Sont bien valorisés les « positionnements intersectifs et les rapports d'empiètement réciproque » (During) dont il a été question dans la première partie.

Pour ALIS, cette convention participe à l'amplification du projet, à sa légitimation, et à la protection des parties impliquées. Dans quelle mesure constitue-t-elle une entreprise de rationalisation, dans une dynamique où la force des désirs, les urgences intellectuelles, sensibles et stratégiques sont prégnants ? Ce n'est pas tant la convention que la volonté de généricité qui représente une potentielle menace.

\footnotetext{
${ }^{9}$ http://i-trace.fr/separation
} 


\section{modalités de collaboration dans La Séparation en 2015}

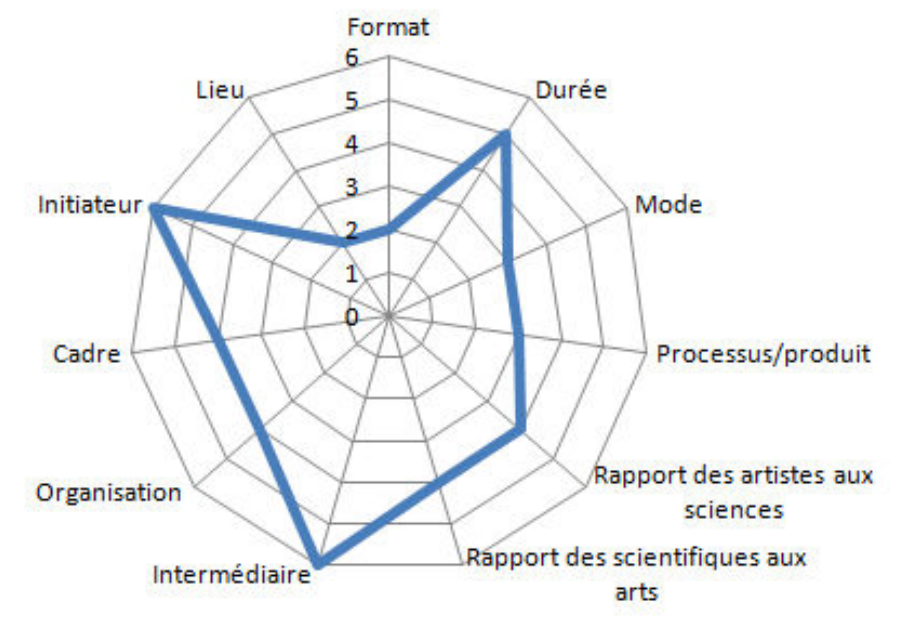

\section{Conclusion}

La nature des collaborations entre chercheurs, ingénieurs et artistes invite à réordonner certains ordres de priorité dans les objectifs poursuivis, en valorisant le processus plutôt que le résultat, mais aussi en mettant en exergue le rôle du prototype comme objet commun aux artistes, aux chercheurs et aux ingénieurs ; objet particulièrement stimulant et capable de donner le change aux attentes institutionnelles. Apparaît également comme crucial le rôle du traducteur ou intermédiaire entre les différents mondes. Celui-ci déplace le projet dans les interstices où il pourra redéfinir l'environnement qui le porte. L'intermédiaire est capable de sortir de la partition des « deux cultures » en jouant souvent le rôle d'un « techno-logue », au sens où il peut porter un discours sur la technique appropriable par chacun des acteurs. Il nous semble par ailleurs évident, à ce stade, qu'étudier une collaboration c'est en étudier les glissements et les latences, s'intéresser aussi à des notions moins objectives comme celles des affinités des personnes en présence, leurs désirs, urgences et intérêts.

La modélisation ébauchée, nécessairement simplificatrice et provisoire, nous paraît avoir toutefois une dimension heuristique et constituer une piste intéressante pour interroger encore plus en profondeur ces collaborations. La prise en compte de leur caractère mouvant telle que nous la plébiscitons appelle peut-être à l'élaboration d'une typologie d'entrée et de sortie de collaboration.

Pourquoi ces modes de collaboration peuvent-ils paraître tout particulièrement féconds actuellement? S'appuyant sur la tradition des ingénieurs-artistes, le croisement entre arts, sciences et technologies est essentiel pour construire une culture dans le contexte technologique contemporain qui est celui du numérique. La confrontation de l'artiste qui explore des possibles et de l'ingénieur ou du chercheur qui construit ou analyse des usages doit contribuer à une culture numérique qui ne soit pas vécue comme un asservissement à la technique mais comme un dépassement continuel de celle-ci. 


\section{Références bibliographiques}

Bardiot,C. (2006). 9 Evenings: Theatre and Engineering, Montréal, Fondation Daniel Langlois, disponible sur : $<$ http://www.fondation-langlois.org/html/f/page.php?NumPage $=572>$

Bardiot, C. et Morris, C. (2006). "Interview With Herb Schneider," in 9 Evenings Reconsidered: Art, Theatre, and Engineering, 1966, Cambridge, MIT List Visual Arts Center, 55-64.

Bouchardon, S. (2014). La valeur heuristique de la littérature numérique, Hermann, collection «Cultures numériques », Paris.

Butcher C. et Broeckmann A., Eds. (2010). The future of the lab, Eindhoven, BALTAN Laboratories. Debatty R. et Grover A. (2011). New Art and Science Affinities, Lulu. com.

Goodyear A. C., "Gyorgy Kepes, Billy Klüver, and American Art of the 1960s: Defining Attitudes Toward Science and Technology," Science in Context, vol. 17, no. 4, pp. 611-635, Dec. 2004.

Los Angeles County Museum of Art et M. Tuchman, Eds., A report on the Art and Technology Program of the Los Angeles County Museum of Art, 1967-1971, Los Angeles, Los Angeles County Museum of Art, 1971.

Lallement M. (2015). L'âge du faire: hacking, travail, anarchie, Paris, Seuil.

Lee P. M. (2004). Chronophobia: on time in the art of the 1960's, Cambridge, MIT Press.

Madlener F., "Prototypes, Entretien avec Élie During," L'étincelle, le journal de la création à l'Ircam, no. 7 , juin 2010.

Odin, F. Thuderoz, C., Eds. (2010). Des mondes bricolés? Arts et sciences à l'épreuve de la notion de bricolage. Presses polytechniques et universitaires romandes.

Snow C. P. (1968). Les deux cultures; suivies de Supplément aux deux cultures, Paris, J.J. Pauvert.

Steiner, P. (2010). "Philosophie, technologie et cognition : état des lieux et perspectives", Intellectica (53/54), 7-40. 